\title{
Patients with anomalous aortic origin of the coronary artery remain at risk after surgical repair
}

Shannon N. Nees, MD, ${ }^{\mathrm{a}}$ Jonathan N. Flyer, MD, ${ }^{\mathrm{a}}$ Anjali Chelliah, MD, ${ }^{\mathrm{a}}$ Jeffrey D. Dayton, MD,

Lorraine Touchette, NP, ${ }^{\mathrm{b}}$ David Kalfa, MD, PhD, ${ }^{\mathrm{c}}$ Paul J. Chai, MD, ${ }^{\mathrm{c}}$ Emile A. Bacha, MD, ${ }^{\mathrm{c}}$ and

Brett R. Anderson, MD, MBA, $\mathrm{MS}^{\mathrm{a}}$

\section{ABSTRACT}

Objectives: Anomalous aortic origin of a coronary artery (AAOCA) from the opposite sinus of Valsalva is a rare cardiac anomaly associated with sudden cardiac death (SCD). Single-center studies describe surgical repair as safe, although medium- and long-term effects on symptoms and risk of SCD remain unknown. We sought to describe outcomes of surgical repair of AAOCA.

Methods: We reviewed institutional records for patients who underwent AAOCA repair, from 2001 to 2016, at 2 affiliated institutions. Patients with associated heart disease were excluded.

Results: In total, 60 patients underwent AAOCA repair. Half of the patients $(\mathrm{n}=30)$ had an anomalous left coronary artery arising from the right sinus of Valsalva and half had an anomalous right. Median age at surgery was 15.4 years (interquartile range, 11.9-17.9 years; range, 4 months to 68 years). The most common presenting symptoms were chest pain $(\mathrm{n}=38 ; 63 \%)$ and shortness of breath $(\mathrm{n}=17 ; 28 \%)$; aborted SCD was the presenting symptom in 4 patients $(7 \%)$. Follow-up data were available for 54 patients $(90 \%)$ over a median of 1.6 years. Of 53 patients with symptoms at presentation, $34(64 \%)$ had complete resolution postoperatively. Postoperative mild or greater aortic insufficiency was present in 8 patients $(17 \%)$ and moderate supravalvar aortic stenosis in $1(2 \%)$. One patient required aortic valve replacement for aortic insufficiency. Two patients required reoperation for coronary stenosis at 3 months and 6 years postoperatively.

Conclusions: Surgical repair of AAOCA is generally safe and adverse events are rare. Restenosis, and even sudden cardiac events, can occur and long-term surveillance is critical. Multi-institutional collaboration is vital to identify at-risk subpopulations and refine current recommendations for long-term management. (J Thorac Cardiovasc Surg 2018;155:2554-64)

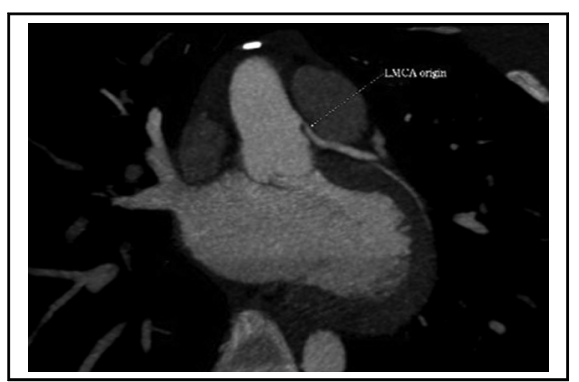

Cardiac CT angiogram in a patient with aborted sudden cardiac death 6 years after unroofing. Coronal view shows the takeoff of the anomalous left coronary artery.

\section{Central Message}

Repair of AAOCA can be performed safely; restenosis, and even sudden cardiac death can occur and long-term surveillance is critical.

\section{Perspective}

Repair of AAOCA can be accomplished safely and can lead to improvement in symptoms and signs of ischemia. Restenosis, and even sudden cardiac events, can occur and long-term surveillance is critical. Multi-institutional collaboration is vital to identify at-risk subpopulations and refine current recommendations for longterm management.

See Editorial Commentary page 2565.
From the a Division of Pediatric Cardiology, Columbia University Medical Center, NewYork-Presbyterian, New York, NY; ${ }^{b}$ Division of Pediatric Cardiology, Weill Cornell Medicine, New York, NY; and ${ }^{\mathrm{c}}$ Division of Cardiac, Thoracic and Vascular Surgery, Columbia University Medical Center, NewYork-Presbyterian Combined Congenital Heart Surgery, New York, NY.

Funding was provided through the Division of Cardiac, Thoracic and Vascular Surgery at Columbia University. Dr Anderson receives salary support through the National Institutes of Health NCATS KL2 TR001874 and NHLBI K23 HL33454. Dr Nees receives salary support through the National Institute of Health award 5T32 HL007854-22.

Read at the 97th Annual Meeting of The American Association for Thoracic Surgery, Boston, Massachusetts, April 29-May 3, 2017.

Received for publication May 1, 2017; revisions received Dec 7, 2017; accepted for publication Dec 24, 2017; available ahead of print March 8, 2018.

Address for reprints: Brett R. Anderson, MD, MBA, MS, Division of Pediatric Cardiology, Department of Pediatrics, Columbia University, 3959 Broadway, 2-N, New York, NY 10032. (E-mail: bra2113@cumc.columbia.edu).

0022-5223/\$36.00

Copyright (c) 2018 by The American Association for Thoracic Surgery

https://doi.org/10.1016/j.jtcvs.2017.12.134
Anomalous aortic origin of a coronary artery (AAOCA) arising from the opposite sinus of Valsalva is a rare cardiac anomaly but is the second most common cause of sudden cardiac death (SCD) in young competitive athletes. ${ }^{1-5}$ The incidence of AAOCA is reported to be

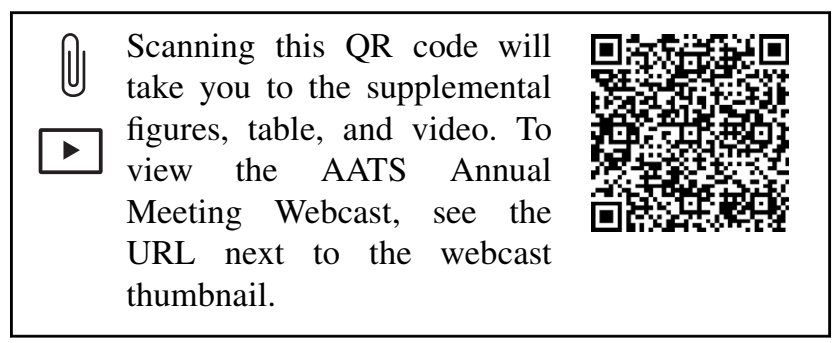




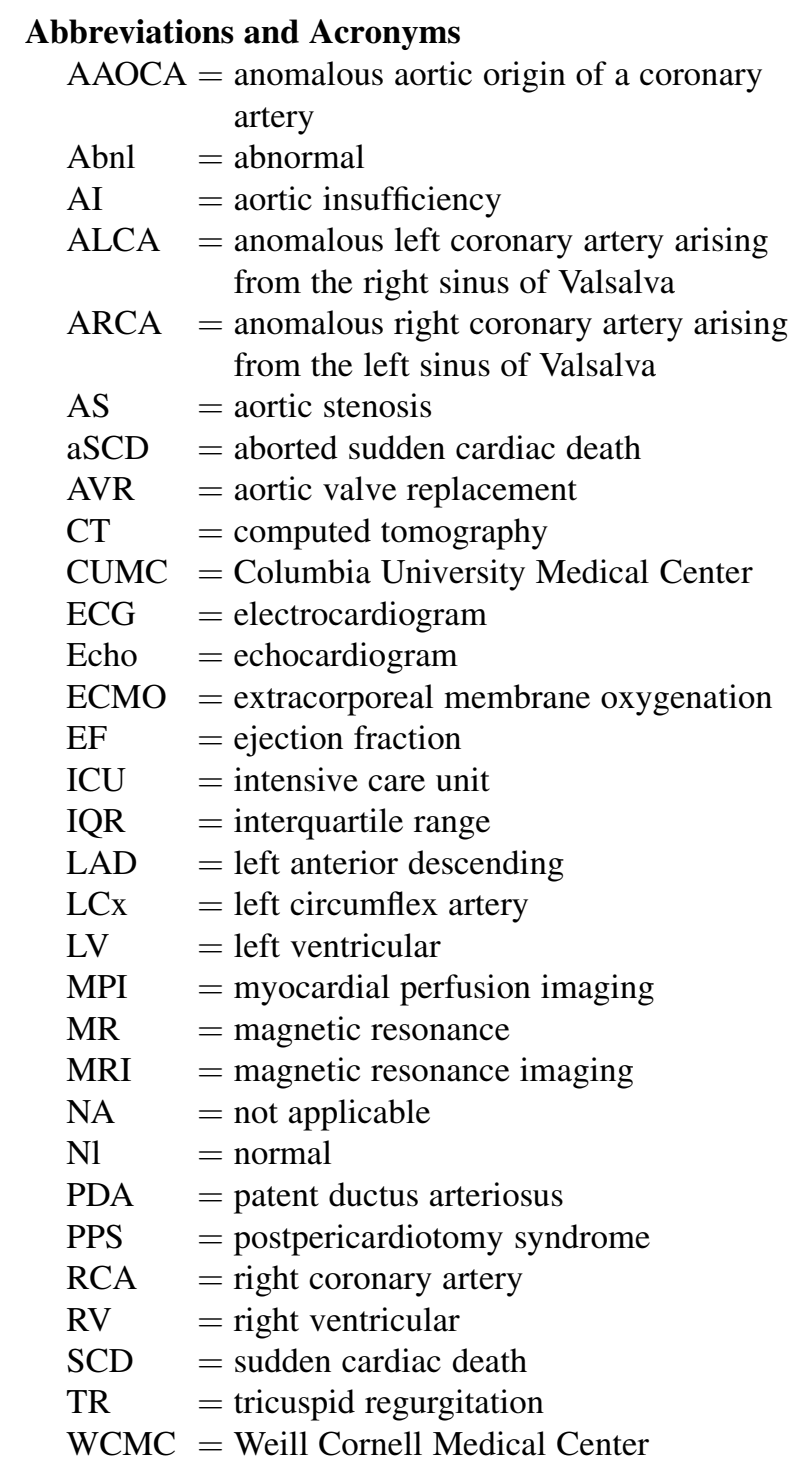

between $0.1 \%$ and $0.7 \%$, with an anomalous right coronary artery arising from the left sinus of Valsalva (ARCA) reported more commonly than an anomalous left coronary artery arising from the right sinus of Valsalva (ALCA). ${ }^{6-8}$

The goal of surgical AAOCA repair is to eliminate the risk of SCD. Single-center studies have shown that surgical repair is generally safe with short-term improvement in symptoms of ischemia after repair. ${ }^{9-15}$ Although a few studies have shown persistent symptoms and/or signs of new ischemia postoperatively, ${ }^{11,15,16}$ data on medium- and long-term outcomes for these patients are limited, and the residual risk of SCD remains unknown. The purpose of this analysis was to describe outcomes after surgical repair of isolated AAOCA at 2 cardiac centers where the same surgical team operates.

\section{METHODS}

\section{Study Population}

We performed a retrospective cohort study of patients who underwent initial surgical repair of AAOCA from 2001 to 2016 at NewYorkPresbyterian/Columbia University Medical Center (CUMC) and New York-Presbyterian/Weill Cornell Medical Center (WCMC). Patients were identified through our institutional surgical database. Attending cardiothoracic surgeons at NewYork-Presbyterian operate at both campuses and report to the Society of Thoracic Surgeons database as a single center. All patients with isolated AAOCA who received surgery by one of our congenital heart surgeons were included, regardless of age. Patients with associated congenital heart defects were excluded.

\section{Perioperative Management and Long-Term Follow-up}

The historical practice at CUMC and WCMC has been to refer all ALCA patients, regardless of symptoms, as well as ARCA patients who show symptoms and/or objective findings of ischemia for surgical repair. The decision to repair asymptomatic ARCA patients has been debated and has remained at the discretion of each cardiologist and cardiothoracic surgeon, in consultation with the patient and family. Repair is typically performed by unroofing of the septum between the aortic lumen and intramural portion of the coronary. In a small subset of patients with short intramural segments, coronary translocation was performed at the discretion of the surgeon. If the intramural portion of the coronary was at or below the level of the aortic commissure, takedown of the tip of the commissure was performed. In patients who required takedown of the aortic commissure, resuspension was attempted in all cases. During the study period, no standardized protocol existed for routine pre- or postoperative followup or testing, and testing was obtained at the discretion of the referring cardiologist and operating surgeon.

\section{Data Collection}

We collected baseline data on patient demographic characteristics, indications for surgery, and type of repair. We examined perioperative and postoperative outcomes. Perioperative outcomes of interest included mechanical circulatory support, arrhythmias, infections, pneumothoraces, and pericardial effusions. Postoperative outcomes of interest included symptoms of ischemia including aborted SCD (aSCD), abnormal electrocardiogram (ECG), echocardiogram (echo), or exercise stress test, new aortic insufficiency (AI), or aortic stenosis (AS), arrhythmia, and reoperation. The ECG, echo, and exercise stress test results were obtained from the most recent available visit.

Most data were obtained via retrospective chart review. For patients who received surgery at CUMC, all patients were also contacted via phone. Verbal consent was obtained to use patients'/parents' subjective reporting of symptoms and vital status. For patients followed by a cardiologist at an outside institution, written consent was obtained to contact the primary cardiologist for follow-up records. Data on patients treated at WCMC were obtained via retrospective chart review with waiver of informed consent. Longitudinal follow-up data had recently been obtained and recorded in the medical records as part of an internal quality improvement effort. In patients for whom no longitudinal follow-up was available, the most recent vital status was obtained using the Social Security death index.

\section{Statistical Methods}

Clinical and demographic variables were described using standard summary statistics. Change in pre- and postoperative symptoms and test data were described graphically. Standard univariable analyses were used to compare pre- and postoperative characteristics including $\chi^{2}$, Fisher exact, unpaired $t$ test, and Kruskall-Wallis test, as appropriate. Analyses were performed using Stata software, version 13.1 (StataCorp, College Station, 
Tex). This study was approved by the respective institutional review boards at CUMC and WCMC.

\section{RESULTS}

\section{Baseline Patient Characteristics}

In total, 60 patients ( 38 male, 22 female) were identified who had surgery for AAOCA, $30(50 \%)$ with ALCA and 30 $(50 \%)$ with ARCA. All patients had an interarterial course and nearly all $(\mathrm{n}=56 ; 93 \%)$ patients had an intramural course of the anomalous coronary, which was confirmed at the time of surgery. Median age at surgery was 15.4 years (interquartile range [IQR], 11.9-17.9 years; range, 4 months to 68 years). Twelve patients were older than 21 years at the time of surgery, 4 with ALCA and 8 with ARCA. Patient characteristics are summarized in Table 1.

Symptoms were present in 53 patients $(88 \%)$ at presentation (Table 1). aSCD was the presenting symptom in 4 patients $(7 \%)$ with ALCA. There were no significant differences between presentation in patients with ARCA and ALCA (Figure 1).

\section{Preoperative Testing}

Of the 58 patients $(97 \%)$ with a preoperative ECG available for review, $13(22 \%)$ were abnormal (Figure E1, A). All but 2 patients $(3 \%)$ had normal ventricular function on preoperative echo. The 2 patients with abnormal function both presented with cardiac arrest (Table E1).

A total of 26 patients $(43 \%)$ underwent exercise stress testing with stress ECG before surgical repair and 17 $(28 \%)$ also had nuclear myocardial perfusion imaging
(Table 2). More patients with ARCA compared with ALCA underwent exercise stress testing $(P<.001)$ and nuclear myocardial perfusion testing $(P<.001)$. Of the 26 patients with exercise testing, $7(27 \%)$ had abnormal ST changes on ECG during the test that could represent ischemia and 6 patients $(23 \%)$ complained of chest pain. On nuclear testing, 7 (41\%) had abnormal perfusion imaging (Figure E1, $B$, and Table E1). Additional imaging including cardiac computed tomography (CT) angiogram, magnetic resonance imaging (MRI), and cardiac catheterization was obtained in 46 patients ( $77 \%$; Table 2 ).

\section{Surgical Repair}

Six surgeons performed the surgeries during the study period. Coronary unroofing was performed in 56 patients $(93 \%)$ and coronary translocation in 4 patients $(7 \%)$. Of the 4 patients who had coronary translocation, 3 did not have an intramural course. In the fourth patient, an unroofing was initially attempted but the intramural portion was short and there was still an acute angle of takeoff on echo; the patient was put back on cardiopulmonary bypass and a translocation was performed. Surgery details are reported in Table 3. There were no reported intraoperative complications and no intraoperative mortalities.

\section{Perioperative Outcomes}

Only 1 patient had a major acute postoperative complication. This patient experienced an out-of-hospital cardiac arrest and was cannulated onto extracorporeal membrane oxygenation preoperatively and required extracorporeal

TABLE 1. Patient characteristics and presentation

\begin{tabular}{|c|c|c|c|c|}
\hline Patient characteristics & All patients & ARCA & ALCA & $P$ value \\
\hline All patients & $60(100)$ & $30(50)$ & $30(50)$ & \\
\hline Male sex & $38(63)$ & $19(63)$ & $19(63)$ & 1.00 \\
\hline Age at surgery in years & $15.4(11.9-17.9)$ & $15.5(13.4-41.0)$ & $15.3(10.8-17.5)$ & .225 \\
\hline Age at last follow-up in years & $16.8(14.5-19.6)$ & $16.4(14.7-19.6)$ & $17.1(14.5-19.2)$ & .986 \\
\hline Weight at surgery, $\mathrm{kg}$ & $60.2 \pm 24.9$ & $62.9 \pm 23.5$ & $57.4 \pm 26.3$ & .400 \\
\hline Symptoms at presentation & $53(88)$ & $28(93)$ & $25(83)$ & .424 \\
\hline Chest pain & $38(63)$ & $22(73)$ & $16(53)$ & .108 \\
\hline Shortness of breath & $17(28)$ & $10(33)$ & $7(23)$ & .390 \\
\hline Palpitations & $12(20)$ & $8(27)$ & $4(13)$ & .197 \\
\hline Syncope & $12(20)$ & $5(17)$ & $7(23)$ & .519 \\
\hline Aborted sudden cardiac death & $4(7)$ & $0(0)$ & $4(13)$ & .112 \\
\hline No symptoms at presentation & $7(12)$ & $2(7)$ & $5(17)$ & .424 \\
\hline Family history & $3(5)$ & $2(7)$ & $1(3)$ & 1.00 \\
\hline Murmur & $2(3)$ & $0(0)$ & $2(7)$ & .492 \\
\hline Abnormal ECG & $1(2)$ & $0(0)$ & $1(3)$ & 1.00 \\
\hline History of PDA & $1(2)$ & $0(0)$ & $1(3)$ & 1.00 \\
\hline
\end{tabular}

Numbers represent means/medians (SD/IQR) for continuous variables and numbers (\%) for categorical variables. ARCA, Anomalous right coronary artery arising from the left sinus of Valsalva; $A L C A$, anomalous left coronary artery arising from the right sinus of Valsalva; $E C G$, electrocardiogram; $P D A$, patent ductus arteriosus. $* P$ for comparison between ARCA and ALCA. 


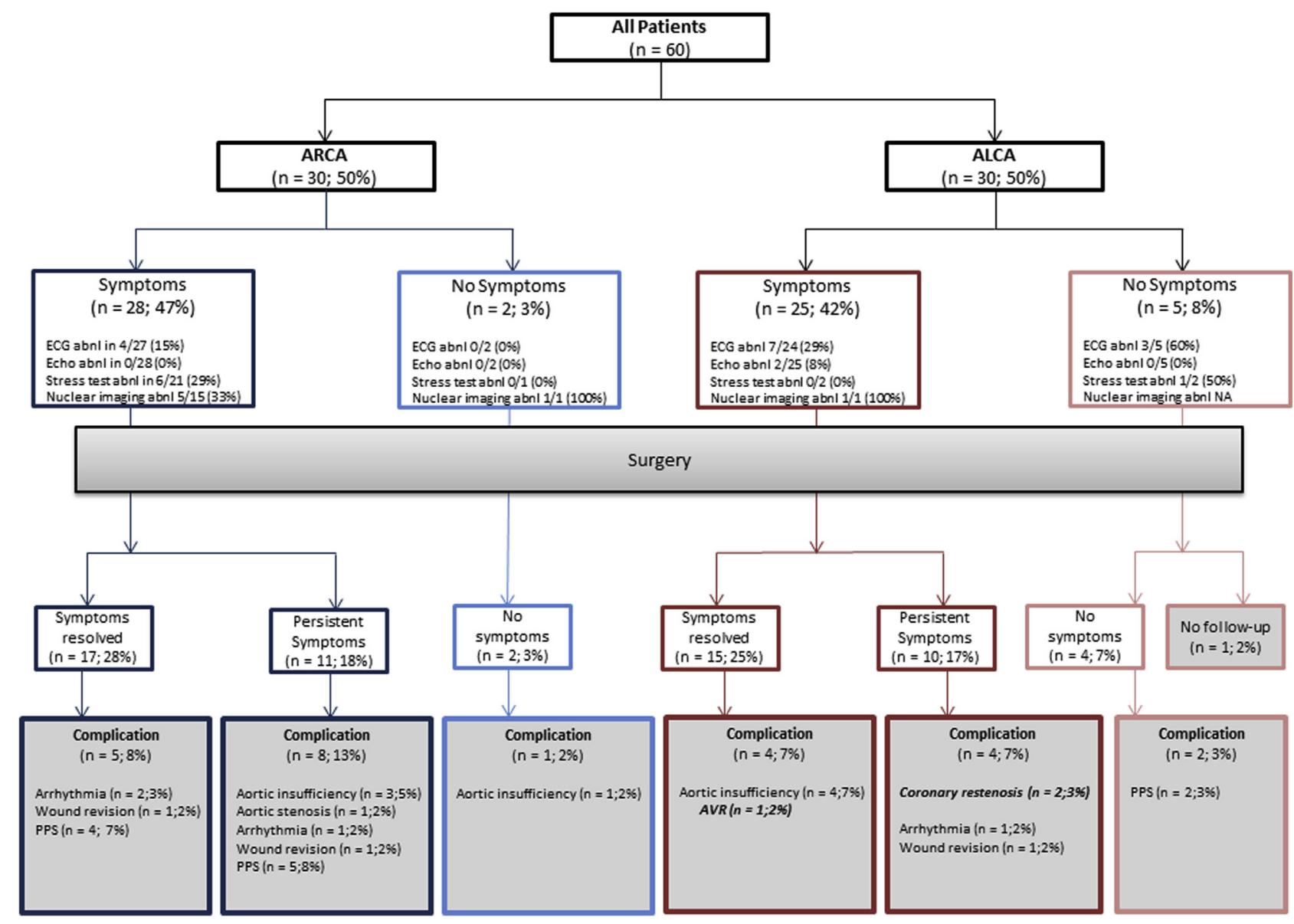

FIGURE 1. Consort diagram for patient outcomes. The outcome for patients on the basis of initial anatomy and symptoms are described. Abnormal echocardiograms include those with abnormal function; all echocardiograms indicated an anomalous coronary. All percentages represent the percent of total patients, $\mathrm{n}=60$. ARCA, Anomalous right coronary artery arising from the left sinus of Valsalva; $A L C A$, anomalous left coronary artery arising from the right sinus of Valsalva; ECG, electrocardiogram; Abnl, abnormal; Echo, echocardiogram; NA, not applicable; PPS, postpericardiotomy syndrome; AVR, aortic valve replacement.

membrane oxygenation postoperatively because of inability to wean from cardiopulmonary bypass. Minor acute postoperative complications occurred in 6 patients $(10 \%)$, including transient arrhythmia requiring treatment $(n=4$; $7 \%)$, superficial wound infection $(\mathrm{n}=1 ; 2 \%)$, and pneumothorax requiring chest tube placement $(\mathrm{n}=1 ; 2 \%)$. After discharge, 11 patients $(18 \%)$ developed postpericardiotomy syndrome at a median of 11 days (IQR, 7-29 days; range, 6-67 days), 2 of whom required pericardiocentesis. Postpericardiotomy syndrome was more common in patients with ARCA compared with those with ALCA $(P=.020$; Table 4$)$. Median length of stay in the intensive care unit (ICU) was 2 days (IQR, 2-3 days; range, 123 days) and median total length of stay was 4 days (IQR, 4-5; range, 2-42 days).

\section{Follow-up}

Symptoms. Longitudinal follow-up data were available from the primary cardiologist for 54 patients $(90 \%)$ over a median of 1.6 years (IQR, 0.6-2.5 years; range, 1 month to 9.4 years). An additional 5 patients $(8 \%)$ responded to phone calls regarding their symptoms but did not complete consent to contact their cardiologist. Of 53 patients with symptoms at presentation, $32(60 \%)$ had complete resolution postoperatively and $9(17 \%)$ had a change in their symptoms (Figure 2). No asymptomatic patients developed symptoms postoperatively.

ECG. On initial postoperative ECG, 32 patients $(53 \%)$ had ST changes. Of 50 patients with postdischarge ECG records available at a median of 583 days (IQR, 179-884 days; range, 13-3360 days), 6 (12\%) had abnormal findings, none of which were ischemic changes (Figure E1, $A$ ).

Echo. Postdischarge echo records were available in 48 patients $(80 \%)$ at a median of 583 days (IQR, 222-874 days; range, $12-3360$ days) postoperatively. Of those, $12(25 \%)$ had abnormal findings, including mild AI $(\mathrm{n}=4 ; 8 \%)$, moderate AI $(\mathrm{n}=3 ; 6 \%)$, and severe AI $(\mathrm{n}=1 ; 2 \%$; Figure 3), moderate supravalvar AS $(n=1 ; 2 \%)$ presumed 
TABLE 2. Pre- and postoperative testing

\begin{tabular}{|c|c|c|c|c|}
\hline Type of testing & All patients & ARCA & ALCA & $P$ value \\
\hline \multicolumn{5}{|l|}{ Preoperative testing } \\
\hline Electrocardiogram* & $58(97)$ & $29(97)$ & $29(97)$ & 1.00 \\
\hline Echocardiogram & $60(100)$ & $30(100)$ & $30(100)$ & 1.00 \\
\hline Exercise stress test & $26(43)$ & $22(73)$ & $4(13)$ & $<.001 \dagger$ \\
\hline Nuclear stress test & $17(28)$ & $16(53)$ & $1(3)$ & $<.001 \dagger$ \\
\hline CT angiogram & $37(62)$ & $22(73)$ & $15(50)$ & .063 \\
\hline MR angiogram & $8(13)$ & $3(10)$ & $5(17)$ & .706 \\
\hline Cardiac catheterization & $12(20)$ & $7(23)$ & $5(17)$ & .519 \\
\hline \multicolumn{5}{|l|}{ Postoperative testing } \\
\hline Electrocardiogram & $50(93)$ & $28(97)$ & $22(88)$ & .326 \\
\hline Echocardiogram & $48(89)$ & $27(93)$ & $21(84)$ & .399 \\
\hline Exercise stress test & $34(63)$ & $19(66)$ & $15(60)$ & .675 \\
\hline Nuclear stress test & $18(33)$ & $11(38)$ & $7(28)$ & .440 \\
\hline CT angiogram & $3(6)$ & $0(0)$ & $3(13)$ & .086 \\
\hline MR angiogram & $4(7)$ & $0(0)$ & $4(16)$ & $.040^{\dagger}$ \\
\hline Cardiac catheterization & $1(2)$ & $0(0)$ & $1(4)$ & .463 \\
\hline
\end{tabular}

Data are presented as $\mathrm{n}(\%)$ except where otherwise noted. ARCA, Anomalous right coronary artery arising from the left sinus of Valsalva; $A L C A$, anomalous left coronary artery arising from the right sinus of Valsalva; $C T$, computed tomography; $M R$, magnetic resonance. *All patients had preoperative electrocardiograms but several patients did not have records available for our review. †Percentages are of patients with follow-up available $(\mathrm{n}=54)$. $\dagger$ Indicates $P$ value $<.05$. All $P$ values are for comparison between ARCA and ALCA.

to be from the aortotomy closure, ventricular wall motion abnormality $(\mathrm{n}=2 ; 4 \%)$, and pericardial effusion $(\mathrm{n}=1$; $2 \%$ ). None of these abnormalities were present on preoperative echo. There was no correlation between commissure takedown and $\mathrm{AI}(P=.074)$.

Exercise testing. A total of 34 patients $(57 \%)$ had an exercise stress test postoperatively, which consisted of a stress ECG at a median of 346 days postoperatively (IQR, 117882 days; range, 38-3360 days). Eighteen of those patients $(30 \%)$ also had nuclear myocardial perfusion imaging. There was no significant difference between patients with and without postoperative stress testing on the basis of type of anomalous coronary $(P=.30)$, age at surgery $(P=.08)$, preoperative symptoms $(P=.22)$, postoperative symptoms $(P=.96)$, or length of follow-up $(P=.70)$. Of the 34 patients with exercise testing, 5 patients $(15 \%)$ complained of chest pain during the study and 2 patients $(6 \%)$ had ST changes indicative of ischemia. Both patients with ST changes had a similarly abnormal preoperative stress test. An additional 2 patients $(11 \%)$ had abnormal nuclear myocardial perfusion imaging. Only 1 patient had a nuclear perfusion scan preoperatively; it was abnormal (Figure E1, $B$ ). None of the patients who complained of chest pain had ischemic changes.

Of the 4 patients with ST changes during exercise study or abnormal nuclear perfusion results, 1 had intermittent palpitations postoperatively and the others remained asymptomatic. The patient with palpitations is restricted from activity, but the remaining patients have been cleared by their cardiologists and are all active.

Reoperation. Reoperation was required in 3 patients $(5 \%)$ with ALCA. One patient developed moderate AI and had worsening left ventricular dilation. This patient required aortic valve replacement 4.7 years after initial surgery. Two patients $(3 \%)$ with ALCA required reoperation for restenosis of the anomalous coronaries. One patient who was 68 years old at the time of initial surgery and received repair because of chest pain with minimal exertion, had recurrent chest pain 2 months after surgery. ECG showed nonspecific ST and T-wave abnormalities. An exercise

TABLE 3. Intraoperative findings and characteristics

\begin{tabular}{|c|c|c|c|c|}
\hline Variable & All patients & ARCA & ALCA & $P$ value \\
\hline \multicolumn{5}{|l|}{ Type of surgical repair } \\
\hline Unroofing & $56(93)$ & $29(97)$ & $27(90)$ & .612 \\
\hline Coronary translocation & $4(7)$ & $1(3)$ & $3(10)$ & .612 \\
\hline Commissure involvement & $16(27)$ & $9(30)$ & $7(23)$ & .559 \\
\hline Cardiopulmonary bypass time in minutes & $55(45-66)$ & $55(45-61)$ & $53(43-74)$ & .827 \\
\hline Aortic cross-clamp time in minutes & $35(26-43)$ & $35(27-43)$ & $32(26-43)$ & .894 \\
\hline
\end{tabular}

Numbers represent median (IQR) for continuous variables and numbers (\%) for categorical variables. ARCA, Anomalous right coronary artery arising from the left sinus of Valsalva; $A L C A$, anomalous left coronary artery arising from the right sinus of Valsalva. $* P$ value for comparison between ARCA and ALCA. 
TABLE 4. Complications

\begin{tabular}{|c|c|c|c|c|}
\hline Complication & All patients & ARCA & ALCA & $P$ value \\
\hline \multicolumn{5}{|l|}{ Acute perioperative complications } \\
\hline Perioperative ECMO and hemorrhage & $1(2)$ & $0(0)$ & $1(3)$ & 1.00 \\
\hline Arrhythmia requiring treatment & $4(7)$ & $4(13)$ & $0(0)$ & .112 \\
\hline Pneumothorax requiring chest tube & $1(2)$ & $1(3)$ & $0(0)$ & 1.00 \\
\hline Superficial wound infection & $1(2)$ & $0(0)$ & $1(3)$ & 1.00 \\
\hline Postpericardiotomy syndrome & $11(18)$ & $9(30)$ & $2(7)$ & $.020^{*}$ \\
\hline \multicolumn{5}{|l|}{ Follow-up complications } \\
\hline Reoperation for coronary stenosis & $2(3)$ & $0(0)$ & $2(7)$ & .492 \\
\hline Aborted sudden cardiac death & $1(2)$ & $0(0)$ & $1(3)$ & 1.00 \\
\hline $\mathrm{AI}+$ & $7(12)$ & $3(10)$ & $4(13)$ & 1.00 \\
\hline Aortic valve replacement for aortic insufficiency & $1(2)$ & $0(0)$ & $1(3)$ & 1.00 \\
\hline Arrhythmia & $4(7)$ & $3(10)$ & $1(3)$ & .612 \\
\hline Wound revision & $3(5)$ & $2(7)$ & $1(3)$ & 1.00 \\
\hline Supravalvar aortic stenosis & $1(2)$ & $1(3)$ & $0(0)$ & 1.00 \\
\hline
\end{tabular}

Data are presented as n (\%) except where otherwise noted. ARCA, Anomalous right coronary artery arising from the left sinus of Valsalva; $A L C A$, anomalous left coronary artery arising from the right sinus of Valsalva; ECMO, extracorporeal membrane oxygenation; $A I$, aortic insufficiency. *Indicates $P<.05$. All $P$ values are for comparison between ARCA and ALCA. †Only mild or greater AI as assessed on echocardiogram was included.

stress test was concerning for ischemia in multiple regions. Significant stenosis was found at the origin of the anomalous left coronary orifice on cardiac catheterization, and coronary bypass grafting was performed 66 days after initial surgery without complication. Another patient was 10 years old at the time of initial surgery and had preoperative symptoms including chest pain with exertion, palpitations, and syncope with exertion. This patient experienced a cardiac arrest during tryouts for a high school sports team 6 years postoperatively. The patient had a negative stress test and reportedly normal echo before being cleared for athletic participation. She did complain of occasional dizziness before the event but denied any previous episodes of chest pain. Postarrest noninvasive imaging using cardiac CT angiogram revealed ostial narrowing with hypoattenuated material at the area of unroofing, which appeared consistent with recurrent fibrosis (Figure E2 and Video 1). Intraoperative examination revealed fibrous tissue around

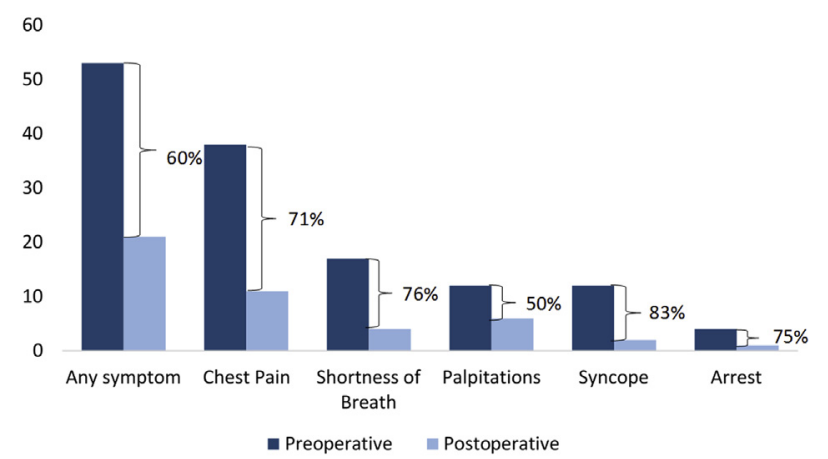

FIGURE 2. Change in symptoms of ischemia. Bar chart depicts the number of patients with symptoms pre- and postoperatively. Brackets represent percent change in symptoms. Includes patients in whom pre- as well as postoperative data were available $(n=59)$. All patients with postoperative symptoms experienced preoperative symptoms. No asymptomatic patients developed symptoms postoperatively. the left coronary orifice, which was resected. The patient continued to have premature ventricular contractions on routine postoperative monitoring; an implantable cardiac defibrillator was placed and the patient was restricted from competitive sports. Both patients with restenosis are asymptomatic at 3 and 1 years after the second operation, respectively.

Other complications. There were 3 patients $(5 \%)$ who required scar revision either for discomfort $(n=2)$ or abnormal healing $(\mathrm{n}=1)$. There were no reported deaths at the time of writing of this report. In patients without follow-up data, this was confirmed using the Social Security death index.

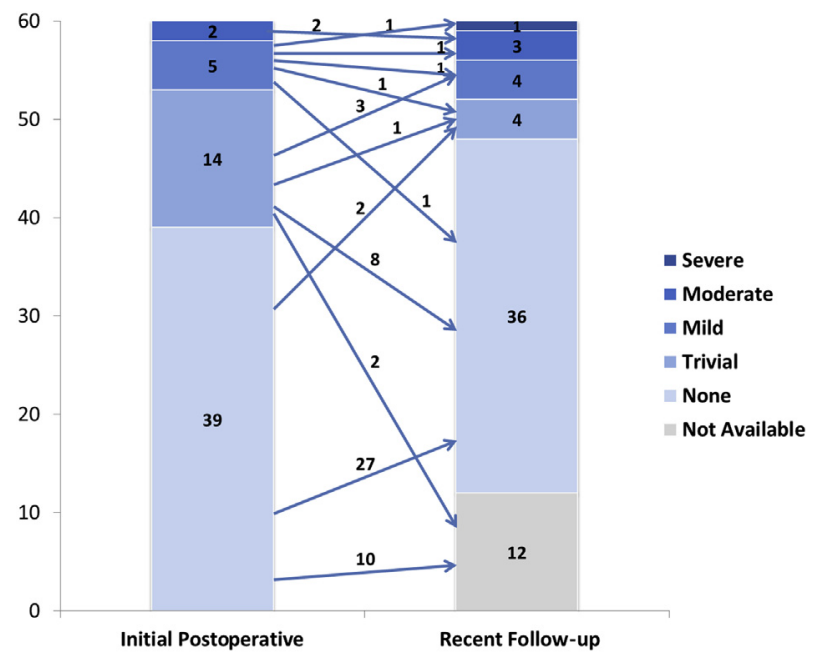

FIGURE 3. Postoperative aortic insufficiency. Bar graphs represent the number of patients with any degree of aortic insufficiency on initial postoperative and most recent follow-up echocardiogram. As noted, there were 12 patients without follow-up echocardiogram available, represented by the light gray box. The median time for follow-up echocardiogram was 583 days postoperatively. 


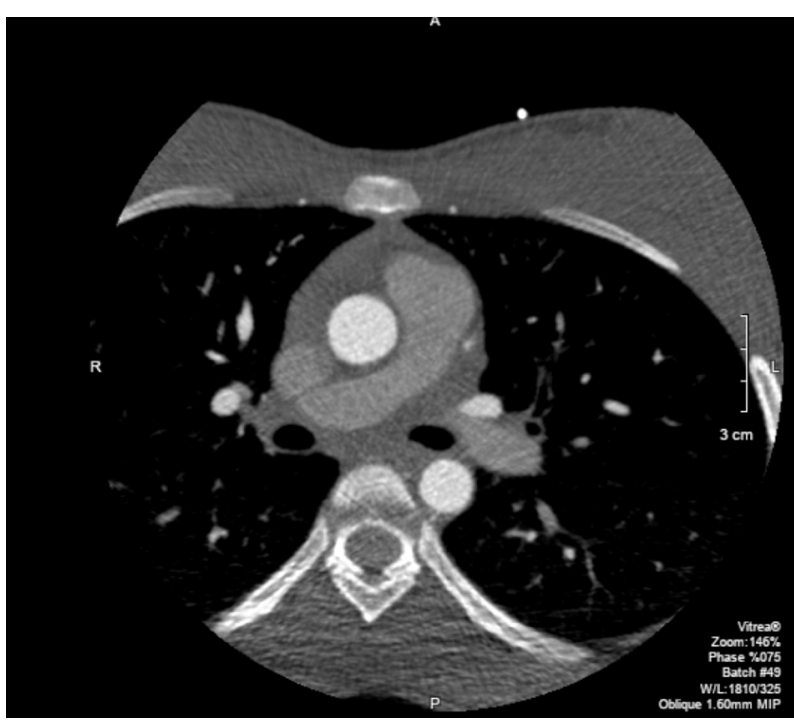

VIDEO 1. Axial multiplanar reconstruction images from cardiac computed tomography performed after cardiac arrest in the patient who underwent unroofing of an anomalous left coronary arising from the right sinus of Valsalva shows a ridge of hypoattenuation at the left coronary origin that extends rightward of the intercoronary commissure, with associated orifice narrowing (indicated by the yellow arrow). Video available at: http://www.jtcvsonline.org/article/S0022-5223(18)30307-6/fulltext.

\section{DISCUSSION}

Surgical repair of AAOCA is generally safe and major acute postoperative complications are rare. In our study, $60 \%$ of patients had resolution of their symptoms postoperatively. However, $40 \%$ of patients still complained of some subjective symptoms although most had normal postoperative testing including exercise tests and ECGs. This might indicate that many of the preoperative symptoms were not, in fact, attributable to the AAOCA and it is unclear whether symptoms are predictive of true ischemia. Recent studies have drawn attention to the possibility that patients with repaired AAOCA might continue to experience not only subjective symptoms, but also objective signs of ischemia. ${ }^{11,15,16}$ Although the theoretical risk was known due to scarring of the coronary ostia, our study is, to our knowledge, the first to report restenosis of an unroofed anomalous coronary and aSCD after surgical repair of AAOCA. Our study also reports risks of supravalvar AS after coronary unroofing and AI even in patients who do not undergo aortic commissural takedown.

Mortality after surgical repair of AAOCA is very rare. A survey of 113 participants from the Congenital Heart Surgeons Society reports only 2 deaths after surgical repair. ${ }^{17}$ However, restenosis of the anomalous coronary can occur. To our knowledge, there is 1 case in the literature of SCD during exercise after surgical repair of AAOCA in a patient who did not have an unroofing, but who underwent ostial plasty and pulmonary artery translocation. ${ }^{18}$ In that patient as well as our own, stress testing was performed before clearing for sports participation and was reportedly normal. Our patient with aSCD presented 6 years after initial repair. Our patient did have some nonspecific dizziness before her arrest, but no evidence of restenosis or ischemia on routine testing. These data highlight the limitations of functional testing in predicting ostial stenosis.

In our second patient with ostial restenosis, chest pain was the presenting symptom. The patient had an abnormal exercise stress test before her second repair, which led to cardiac catheterization. This patient presented within weeks of initial surgery and was in her $60 \mathrm{~s}$, thus the mechanism of restenosis in this patient might have been different than in the first patient described - either because of postoperative changes or underlying coronary artery disease and abnormal healing of the surgical repair, rather than fibrous scarring over time.

Brothers et al reported that $38 \%$ of patients with AAOCA repair had evidence of ischemia on postoperative testing with patent coronary ostia on echo, whereas only 1 had evidence of ischemia preoperatively, perhaps indicating that surgery itself might cause myocardial changes or that these changes might develop over time regardless of the surgery. ${ }^{16}$ In one report by Mainwaring et al, of 115 patients who underwent surgical repair of AAOCA, 2 required reoperation for persistent ischemia, 1 for revision of the initial repair, and 1 for a myocardial bridge. However, in these patients, anatomic issues were identified that were likely to contribute to the postoperative ischemia, including a narrow coronary orifice and myocardial bridging. ${ }^{15}$ In our study, no patients developed new ischemic changes on postoperative ECG and 1 patient had abnormal myocardial perfusion imaging with no baseline for comparison. Three patients showed persistent abnormalities consistent with ischemia on exercise stress testing or stress myocardial perfusion imaging. Although these patients all had patent coronary ostia on echo, perhaps these patients deserve further imaging to better define the coronary ostia and coronary perfusion postoperatively.

On the basis of the risk of postoperative complications, all patients who undergo surgery for AAOCA should have lifelong cardiovascular care, but postoperative follow-up is not yet standardized and it is unclear what testing is predictive of adverse events. Considering the available data, it is difficult to determine which patients are at highest risk. Larger studies, such as those currently being conducted by the Congenital Heart Surgeons Society, are needed to risk-stratify postoperative patients. Several institutions, including our own, have worked to develop standardized management protocols for evaluating and managing AAOCA longitudinally, in an effort to track and study outcomes systematically. Brothers and colleagues recently published guidelines recommending routine ECGs and echos and an exercise stress test with myocardial perfusion imaging 3 months postoperatively. ${ }^{19}$ They also recommend cardiac MRI scans 6 months after surgery to assess for changes in the coronary anatomy or myocardial scarring. ${ }^{19}$ 
Current guidelines suggest that asymptomatic patients with normal stress tests can be cleared for exercise 3 months after surgery if there is no history of aSCD and 12 months after surgery if there is a history of aSCD. ${ }^{19,20}$ Our patient who arrested postoperatively met criteria for exercise participation under these guidelines. An MRI scan was not performed because it was not part of that cardiologist's practice. The postarrest CT scan did show narrowing of the coronary ostium. It is not known at what point these changes developed. It stands to reason that if one is creating and manipulating a neo-ostium surgically, some scar will form, eventually leading, in some cases, to restenosis. It is possible that patients would benefit from MRI or CT screening at regular intervals and that advanced imaging should be incorporated into follow-up. Families should be counseled that even after patients are cleared for exercise, they remain at risk for ischemic complications and that it is critically important that first responders trained in cardiopulmonary resuscitation and equipped with an automated external defibrillator be present.

The incidence of more minor complications in our study was within the previously reported range- $-10 \%$ to $67 \% .^{9,11,12}$ This wide range is likely because of different definitions and methods of data collection rather than from significant differences among centers. Our study confirms that the risk of postoperative AI, leading to a reoperation in 1 case, is not trivial (17\% in our cohort vs $21 \%$ in previously reported populations) and might be even higher because $20 \%$ of our patients did not have postoperative echo results available. ${ }^{11}$ Other studies have also reported low rates of aortic valve replacement secondary to AI. ${ }^{9,10}$ Postoperative AI is thought to be a result of commissural takedown. However, our study suggests that there might be more complex issues at play because 3 of the 7 patients who developed postoperative AI did not have takedown of the aortic commissure during their surgery and one of them underwent coronary translocation alone, without unroofing. This raises the possibility of a structural deficiency of the aortic wall at the level of the commissure/crossing intramural coronary. Indeed, the one patient who required aortic valve replacement was found to have extra laxity of the portion of the wall that carried the commissure. The commissure was well healed after the takedown and resuspension, but because of this laxity, was able to prolapse into the aortic lumen. On the basis of our data, one surgeon has modified his technique during coronary unroofing to minimize disruption of the aortic valve by fenestrating the intramural tunnel as opposed to splaying it open from beginning to end.

One of our patients developed postoperative supravalvar AS, thought to be due to the aortotomy closure. To our knowledge, this complication has not been previously reported. Other complications cited in the literature include ventricular dysfunction, heart block, and other arrhythmias and postpericardiotomy syndrome. ${ }^{9,17,21}$

There are some notable limitations of this study. As a retrospective review of surgical outcomes, it is subject to inherent bias, including selection bias on the basis of the types of patients who were referred for surgery and underwent more extensive pre- and postoperative testing. We lacked a nonsurgical control cohort to compare long-term outcomes, which limits our ability to accurately assess the magnitude of risk reduction or to opine on the decision to operate. We included patients of all ages, including 12 patients older than 21 years of age. These patients might have subclinical acquired coronary artery disease in addition to their coronary anomaly that affects outcomes. However, patients with AAOCA who have already survived to older age might be at lower risk for SCD than those who have not already proven that they can survive.

The true incidence of restenosis and SCD after repair is difficult to determine from this size of cohort and it is also difficult to determine what types of postoperative testing are most useful for predicting adverse outcomes. Multicenter studies on postoperative testing and outcomes, such as the Congenital Heart Surgeons Society AAOCA registry, will be critical in determining the frequency and predictors of postoperative SCD with greater precision. It is notable that in our series all serious complications occurred in patients with ALCA (ie, in patients who had an absolute indication for surgery). It will be important to see whether this remains true in a larger cohort. If so, this might suggest that more aggressive postoperative followup is warranted in patients with repaired ALCA.

\section{CONCLUSIONS}

Repair of AAOCA can be accomplished with relatively few complications and can lead to improvement in symptoms and signs of ischemia. Restenosis, and even SCD, can occur and long-term surveillance is critical. Multiinstitutional collaboration is vital to identify at-risk subpopulations and refine current recommendations for long-term management and best practices for follow-up imaging.

\section{Webcast}

You can watch a Webcast of this AATS meeting presentation by going to: https://aats.blob.core.windows.net/ media/17AM/2017-05-02/RM312/05-02-17_Room312_ 1420_Nees.mp4.

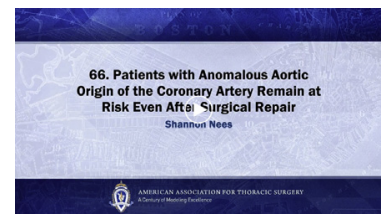




\section{Conflict of Interest Statement}

Authors have nothing to disclose with regard to commercial support.

\section{References}

1. Eckart RE, Scoville SL, Campbell CL, Shry EA, Stajduhar KC, Potter RN, et al Sudden death in young adults: a 25 -year review of autopsies in military recruits. Ann Intern Med. 2004;141:829-34.

2. Maron BJ, Doerer JJ, Haas TS, Tierney DM, Mueller FO. Sudden deaths in young competitive athletes analysis of 1866 deaths in the United States, 1980-2006. Circulation. 2009;119:1085-92.

3. Cheitlin MD, De Castro CM, McAllister HA. Sudden death as a complication of anomalous left coronary origin from the anterior sinus of valsalva: a not-so-minor congenital anomaly. Circulation. 1974;50:780-7.

4. Taylor AJ, Byers JP, Cheitlin MD, Virmani R. Anomalous right or left coronary artery from the contralateral coronary sinus: "high-risk" abnormalities in the initial coronary artery course and heterogeneous clinical outcomes. Am Heart J. 1997;133:428-35.

5. Taylor AJ, Rogan KM, Virmani R. Sudden cardiac death associated with isolated congenital coronary artery anomalies. J Am Coll Cardiol. 1992;20:640-7.

6. Tuo G, Marasini M, Brunelli C, Zannini L, Balbi M. Incidence and clinical relevance of primary congenital anomalies of the coronary arteries in children and adults. Cardiol Young. 2013;23:381-6.

7. Davis JA, Cecchin F, Jones TK, Portman MA. Major coronary artery anomalies in a pediatric population: incidence and clinical importance. J Am Coll Cardiol. 2001;37:593-7.

8. Angelini P, Shah NR, Uribe CE, Cheong BY, Lenge V, Lopez JA, et al. Novel MRI-based screening protocol to identify adolescents at high risk of sudden cardiac death. J Am Coll Cardiol. 2013;61:E1621.

9. Romp RL, Herlong JR, Landolfo CK, Sanders SP, Miller CE, Ungerleider RM, et al. Outcome of unroofing procedure for repair of anomalous aortic origin of left or right coronary artery. Ann Thorac Surg. 2003;76:589-96.

10. Fabozzo A, DiOrio M, Newburger JW, Powell AJ, Liu H, Fynn-Thompson F, et al. Anomalous aortic origin of coronary arteries: a single center experience. Semin Thorac Cardiovasc Surg. 2016;28:791-800.

11. Wittlieb-Weber CA, Paridon SM, Gaynor JW, Spray TL, Weber DR, Brothers JA. Medium-term outcome after anomalous aortic origin of a coronary artery repair in a pediatric cohort. J Thorac Cardiovasc Surg. 2014;147:1580-6.

12. Frommelt PC, Sheridan DC, Berger S, Frommelt MA, Tweddell JS. Ten-year experience with surgical unroofing of anomalous aortic origin of a coronary artery from the opposite sinus with an interarterial course. J Thorac Cardiovasc Surg. 2011;142:1046-51.

13. Davies JE, Burkhart HM, Dearani JA, Suri RM, Phillips SD, Warnes CA, et al. Surgical management of anomalous aortic origin of a coronary artery. Ann Thorac Surg. 2009;88:844-8.

14. Mumtaz MA, Lorber RE, Arruda J, Pettersson GB, Mavroudis C. Surgery for anomalous aortic origin of the coronary artery. Ann Thorac Surg. 2011;91:811-5.

15. Mainwaring RD, Murphy DJ, Rogers IS, Chan FP, Petrossian E, Palmon M, et al. Surgical repair of 115 patients with anomalous aortic origin of a coronary artery from a single institution. World J Pediatr Congenit Hear Surg. 2016;7:353-9.

16. Brothers JA, McBride MG, Seliem MA, Marino BS, Tomlinson RS, Pampaloni $\mathrm{MH}$, et al. Evaluation of myocardial ischemia after surgical repair of anomalous aortic origin of a coronary artery in a series of pediatric patients. J Am Coll Cardiol. 2007;50:2078-82.

17. Brothers J, Gaynor JW, Paridon S, Lorber R, Jacobs M. Anomalous aortic origin of a coronary artery with an interarterial course: Understanding current management strategies in children and young adults. Pediatr Cardiol. 2009;30:911-21.

18. Nguyen AL, Haas F, Evens J, Breur JM. Sudden cardiac death after repair of anomalous origin of left coronary artery from right sinus of Valsalva with an interarterial course: case report and review of the literature. Neth Heart J. 2012;20: 463-71.

19. Brothers JA, Frommelt MA, Jaquiss RD, Myerburg RJ, Fraser CD, Tweddell JS. Expert consensus guidelines: anomalous aortic origin of a coronary artery. $J$ Thorac Cardiovasc Surg. 2017;153:1440-57.

20. Van Hare GF, Ackerman MJ, Evangelista JK, Kovacs RJ, Myerburg RJ, Shafer KM, et al. Eligibility and disqualification recommendations for competitive athletes with cardiovascular abnormalities: task force 4: congenital heart disease: a scientific statement from the American Heart Association and American College of Cardiology. Circulation. 2015;132:e281-91.
21. Penalver JM, Mosca RS, Weitz D, Phoon CK. Anomalous aortic origin of coronary arteries from the opposite sinus: a critical appraisal of risk. BMC Cardiovasc Disord. 2012;12:83.

Key Words: anomalous coronary artery, congenital heart disease, congenital heart surgery, sudden death, pediatrics

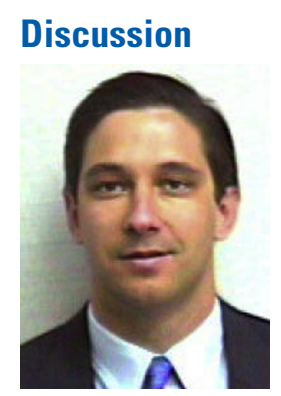

Dr R. Mosca (New York, NY). Shannon, thank you for a very nice presentation of a complex subgroup of patients. The therapy of anomalous aortic origin of the coronary artery is going to be the subject of entire symposia in the future, but for today, just a couple of comments and specific questions on the data that you offered in your paper.

The interest in these anomalies has peaked because they are very perplexing. Although we are aware of the risk of sudden death; the true prevalence, the significance of symptoms, our ability to determine the preoperative anatomic characteristics, the surgical intervention and its effects on postoperative symptoms, and the true risk of postoperative sudden death are still all uncertain.

Your paper attempts to provide further insight into some of these questions. Your survival rates were excellent, yet complications, as you told us, were not infrequent. One of the most important contributions of this paper might be the realization, as others have told us, that we are not entirely sure our operations are preventing postoperative ischemia. At a median follow-up of 19 months, you had 3 patients that needed reoperation, 2 for ischemia, both secondary to obstruction at the reconstructed coronary orifice. That's a $3 \%$ postoperative incidence of ischemia, which is not insignificant, and might actually outweigh the risk of sudden cardiac death in all patients who have these anomalies, because we don't know the underlying prevalence.

My first question is regarding your method of diagnosis. All of us seem to think that the "intramurality," as Marshall quoted, is very important, and nearly all of your patients were described as having intramural coronaries. Can you tell me how that was diagnosed and were you able to validate it in the operating room?

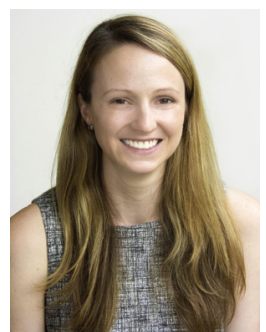

Dr S. N. Nees (New York, NY). The percentage of patients in our study that we quoted as intramural was all based on operative reports. We found very inconsistent data in preoperative echocardiograms in terms of whether they reported the length or presence of an intramural course, and then we didn't specifically look at how that correlated with the intraoperative findings. 
There was no standard practice for pre- or postoperative imaging at our institution during the course of this study, and so a couple of patients had preoperative CTs or MRIs but it wasn't often enough that we could look at a correlation.

Dr Mosca. I would go even further to say that not only is echo not all that good at determining the intramural nature of the coronary but we need to further refine our MRIs and CTAs before we can be absolutely sure that they are defining the intramural nature of these coronaries accurately.

Marshall talked a bit about symptoms, so I will just jump to the punch line here. You had patients who were symptomatic who became asymptomatic, you had some that remained symptomatic after surgery, and some of these patients underwent stress tests. Were they stress echos or stress ECGs?

Dr Nees. They were all exercise stress tests with ECGs and a subset of those patients had nuclear stress imaging as part of their stress test.

Dr Mosca. Given the sporadic nature of the symptoms, the fact that they don't reliably go away and the fact that they don't correlate very well with our postop tests, would you agree that the symptoms, perhaps other than syncope, aren't all that useful in predicting who needs surgery or who is going to do well after surgery?

Dr Nees. I would agree. One limitation of our study is that we didn't have a nonoperative cohort to compare, but it does seem in reviewing the literature and in our study as well that symptoms are subjective. And, as Dr Jacobs mentioned, chest pain is a very frequent symptom in teenage patients who present to our cardiologist's office, and I think it is very difficult to tell what of that is ischemic chest pain versus nonischemic chest pain.

Dr Mosca. I agree. I think we are all learning that.

Fifteen percent of patients had at least mild AI, which for the paper I think you might have to clarify a little bit better, but only required AVR, which you said was unrelated in your article to takedown of the commissure and you suggested might be due to structural abnormality of the aortic wall, which it might be. I would propose, however, that it is likely due to all our manipulations of the aorta in the area of the commissure, takedown, unroofing, mobilizing, especially if the coronary lies close to the commissure or where the leaflet joins the aortic wall. Postdischarge echos weren't available in approximately $20 \%$ of patients, so it's possible it's even higher than you report.

Dr Mosca. What are you doing now to try to avoid postoperative aortic insufficiency when you operate on these patients?

Dr Nees. I'm not a surgeon so I'm not operating, but I think it was interesting in our paper, which were data that I didn't present, that of the 7 patients with at least mild
AI, only 4 of them had takedown of the commissure intraoperatively. So there is clearly something else going on in these patients that's leading to some of the AI, which might be due to the manipulation, and that creates some laxity within the aortic wall and an opportunity for the valve to prolapse. I don't know if our team has changed their surgical management to prevent that.

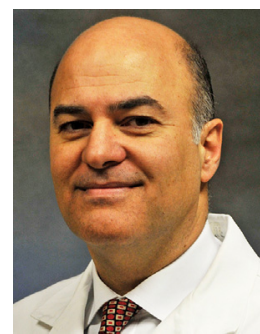

Dr E. Bacha (New York, NY). Very quickly, it was very interesting yesterday from Texas Children's that I think they are avoiding commissural takedown at all costs. We are kind of moving this way as well having seen our fairly high incidence of AI. I don't know that I would always avoid it, I'm not sure how to always avoid it, but I definitely try to stay away from doing anything to disrupt the commissural architecture.

Dr Mosca. Thank you, and I would agree, but sometimes it's very difficult, because the coronary can have only a short intramural segment and exit the aortic wall right at the commissure.

So now in closing I would like to add a comment. Thanks for presenting this and keeping us aware of the potential consequences of our interventions. I would submit, as Dr Jacobs has shown us, that we have a lot to learn about this lesion and thus we might not be applying our surgical procedures appropriately. The vast majority of patients with these anomalies remain undiagnosed and live normal lives, so we have to be mindful of the potential negative consequences of our surgical interventions.

As our imaging modalities uncover these abnormalities, we need to better understand which subsets of patients will benefit from surgery. Otherwise we run the risk of creating more problems than we are actually solving. So multicenter trials, as we are having done, are needed. Unfortunately I believe given the nature of the processes involved we might need decades to draw definitive conclusions. Thank you very much for your excellent presentation.

Dr Nees. Thank you. I agree with your comment.

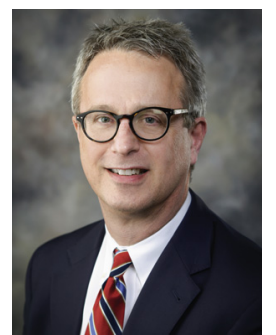

Dr R. D. B Jaquiss (Dallas, Tex). I don't have much to add, except that I would like to introduce a term for us to think about. Before I do that, I would like to congratulate you and the entire Columbia group for presenting a series admitting your flaws. We don't do a lot of that and we should do more of it.

The term that I want us to be thinking about or I think we should begin to think about is number needed to treat, and it's a different way of saying what Ralph said. He said it more clearly with a slightly different accent. But number needed to treat to do some good is one thing, and I don't know if we will ever get that, because we don't really 
know what the risk is, but we can probably get to, and I think this is probably the greatest value of the registry, Marshall, number needed to treat to cause harm. So we know that at least in New York, as you have done it recently, that you need to treat 45 patients to cause 1 aortic valve replacement, perhaps. You need to treat 45 patients to cause 3 cases of postpericardectomy syndrome, which, in somebody who is unoperated is pretty hard to get. And we will hear of deaths in surgically treated patients. Some of them will be the wrong surgery, and there was a miraculous save that Carlos Mery talked about yesterday in somebody with bridging. But I think that until we know how many people we have to treat before we hurt somebody or one of our patients dies, we have to be very circumspect and do that thing which is hardest for surgeons, and that is to not operate at all.

Anyway, I congratulate you on a great paper.

Dr Nees. Thank you very much.

Dr C. Baird (Boston, Mass). Very nice presentation. I have a comment and 2 questions. One comment was about the AR. We have found it to be extremely important that when taking down the commissure to tack it back up. We didn't do that early in our experience and had AR as well.

First question is, are you anticoagulating these patients and if so how are you anticoagulating them and with what agents (anti-platelet or Coumadin) and how are they monitored? What is the timing of starting and ending anticoagulation?

And the second question is regarding postoperative follow-up. You alluded to it in the end but you didn't give us any details. Are you getting CT images before discharge, within a month, 6 months, a year?

Dr Nees. To your first question, the anticoagulation was only ever with aspirin. Most patients were discharged on aspirin but actually not all, and there was some era effect to that, and none of the patients went home on Coumadin or other anticoagulation. And how long you continue the patients on aspirin was at the discretion of the primary cardiologist, so that was variable as well.

To your second question on how we are monitoring these patients postoperatively, that is still something that we are developing, and we are working at Columbia to develop a standardized protocol for follow-up of these patients as they have done at several other institutions. The most recent recommendations from the AATS, which were published earlier this year, did recommend MRI as part of the follow-up, which was not standard in our patients.

Currently, of these 60 patients that were followed, many of them were followed by cardiologists outside of our institution, and so the practices in terms of stress testing as well as imaging were extremely variable. Part of what we are doing as the next step is to try to standardize that.

Dr Baird. We are now leaning toward CT early in the postoperative period.

And then just a final question. Do you do troponins postoperatively and if you do what do they mean?

Dr Nees. That is also somewhat variable. I looked at it as part of the data but didn't end up reporting it, because most of our pediatric patients were treated in our pediatric ICU and had postoperative troponins, and we looked at the peak troponin in those patients and it didn't really seem to correlate with anything. We didn't do statistical analysis but just sort of a subjective look at it. Most of our adult patients who are treated in our adult cardiac ICU did not get postoperative troponins, interestingly. I don't have any insight into what that means.

Dr Baird. Great. Nice presentation.

Dr Nees. Thank you.

Dr Fraser (Houston, Tex). Unfortunately you have confirmed what we are all afraid of, and that is that in very capable hands patients are presenting with ostial stenoses after we have manipulated an ostium. So I have to put you on the spot a little bit. So the next anomalous left that presents to Morgan Stanley with ischemia or syncope that you unroof and then postoperatively you demonstrate that you have achieved the results and early on you don't have provocative concerns, are you going to allow those patients to go back and have unlimited activity? What are you going to do?

Dr Nees. I think the answer is yes, that I would allow them, but I do think that serial imaging is important. And of note, the patient who had the aborted sudden cardiac death did have a nuclear stress test sort of early postoperatively, several years before the arrest, but an exercise stress test, just ECG exercise stress test, very soon before the arrest. I think one of the issues is that we don't really know what provocative testing is most predictive. And she did not have any axial imaging; she had not had a $\mathrm{CT}$ or an MRI in that period. And so I hope that potentially coming up with a protocol that includes axial imaging as well as provocative testing might help us to better identify these patients. But I think the goal of repairing these patients is to allow them to exercise, and it's very hard to limit. This particular patient was very type A, wanted to exercise, wanted to be on the varsity team, and I think it's hard when that patient is in your office to say no when you have no objective evidence that it's unsafe.

Dr Fraser. Very difficult to say no. Thanks very much.

Dr Nees. Thank you. 

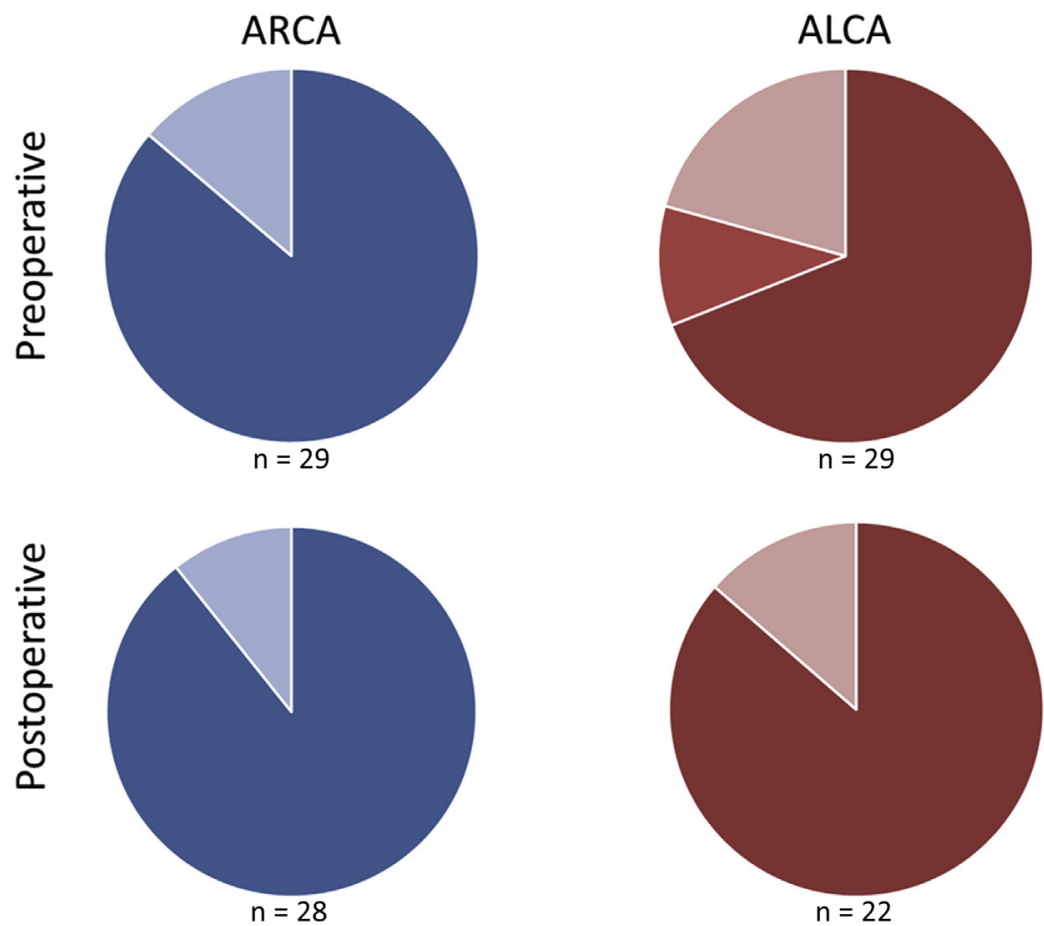

A $\quad$ Normal ECG $\|$ Ischemic changes $\|$ Other abnormality

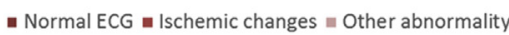
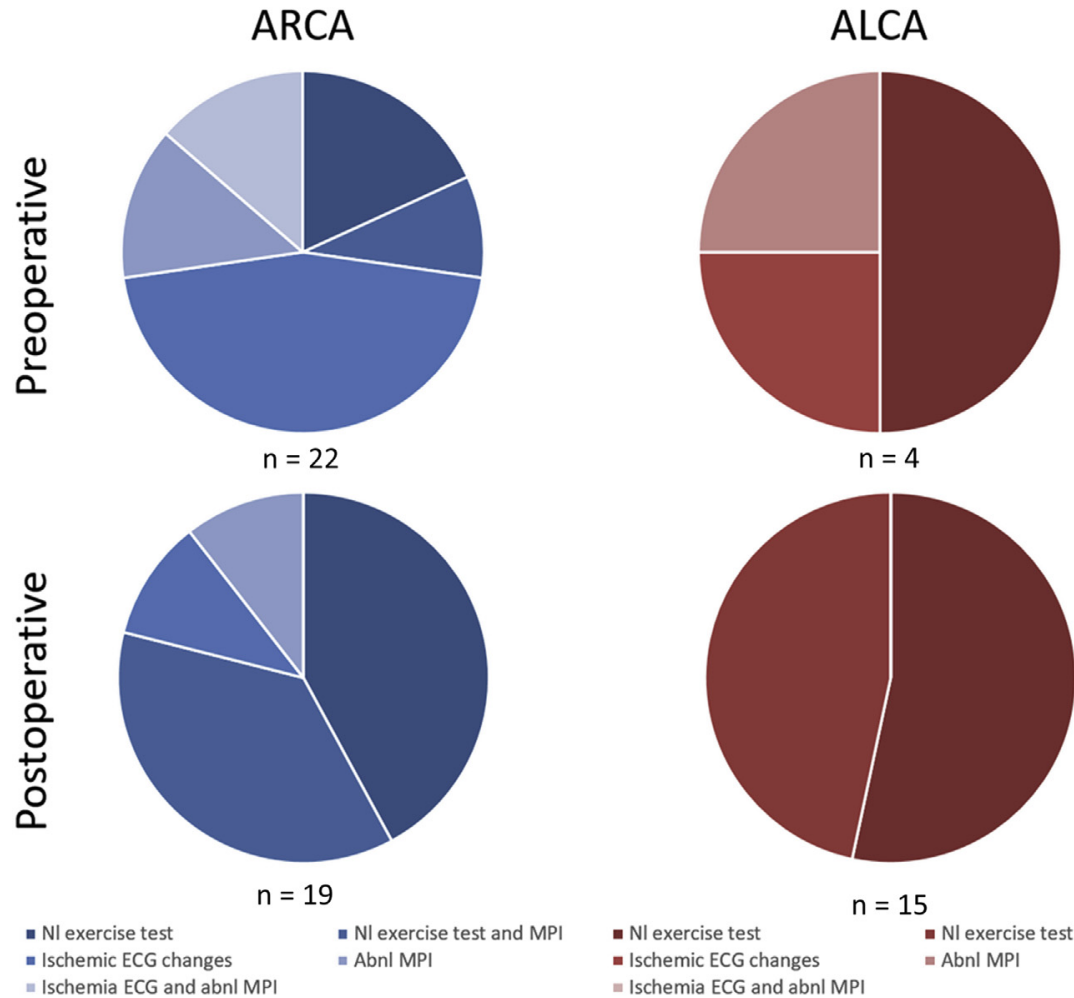
$\mathrm{n}=15$
- Ischemic ECG changes
= Ischemia ECG and abnl MPI

FIGURE E1. Pre- and postoperative testing. Pre- and postoperative electrocardiogram (A) and exercise stress test (B) results. ARCA, Anomalous right coronary artery arising from the left sinus of Valsalva; $A L C A$, anomalous left coronary artery arising from the right sinus of Valsalva; $E C G$, electrocardiogram; $N l$, normal; $M P I$, myocardial perfusion imaging; $A b n l$, abnormal. 

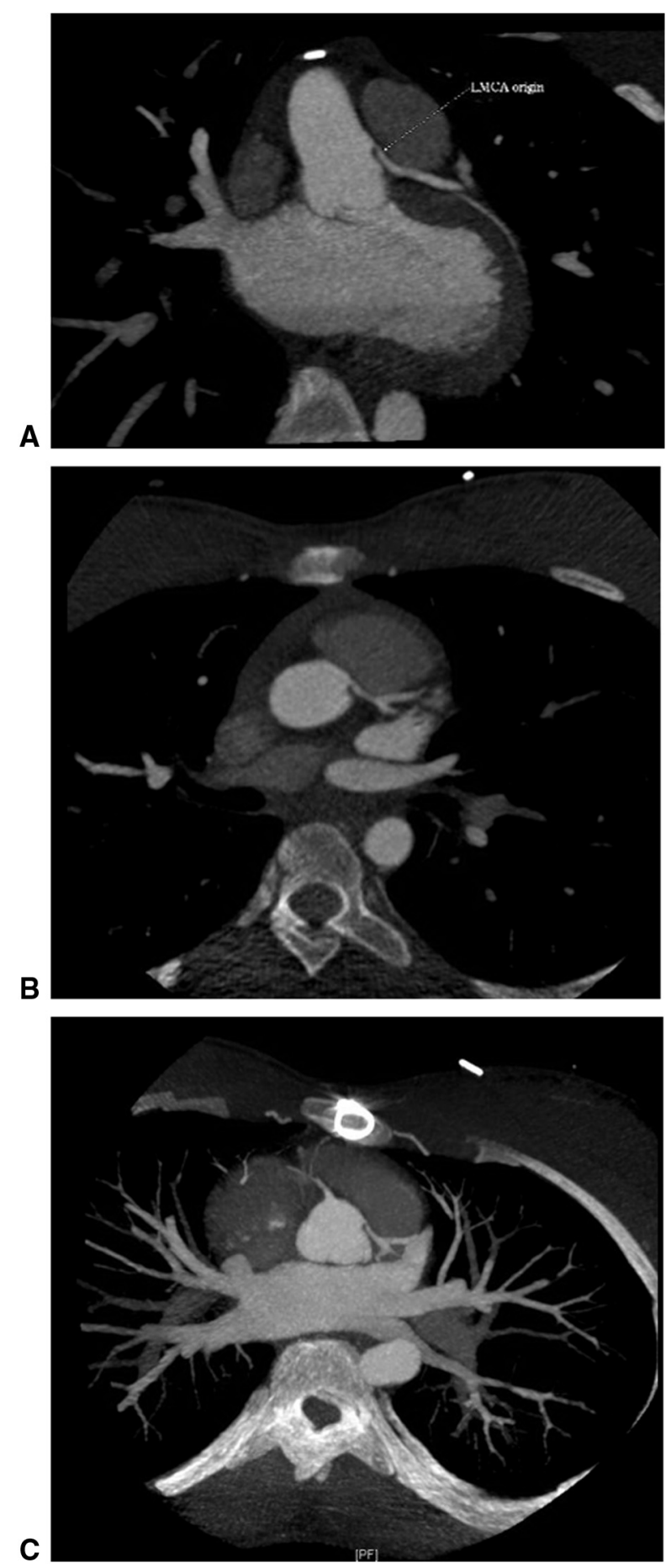

FIGURE E2. Cardiac computed tomography angiogram in a patient with aborted sudden cardiac death 6 years after coronary artery unroofing. A, Coronal view showing the takeoff of the anomalous left coronary artery. $\mathrm{B}$, Axial view showing hypoattenuated material at the site of the coronary unroofing. C, Axial view showing narrowing of the anomalous left coronary artery origin. 
TABLE E1. Preoperative testing abnormalities

\begin{tabular}{|c|c|c|c|}
\hline Study & Patient number & Anomalous coronary & Abnormality \\
\hline \multicolumn{4}{|c|}{ Echocardiogram } \\
\hline & 19 & Left & EF $25 \%$, severely decreased LV function after cardiac arrest \\
\hline & 23 & Left & Moderately decreased RV and LV function, moderate TR after cardiac arrest \\
\hline \multicolumn{4}{|c|}{ Electrocardiogram } \\
\hline & 1 & Left & LV hypertrophy \\
\hline & 4 & Left & LV hypertrophy \\
\hline & 7 & Left & LV hypertrophy \\
\hline & 13 & Left & LV hypertrophy \\
\hline & 19 & Left & T-wave changes consistent with anteroseptal infarct \\
\hline & 20 & Right & LV hypertrophy \\
\hline & 21 & Right & Left axis deviation \\
\hline & 22 & Left & LV hypertrophy \\
\hline & 23 & Left & Low voltage with deep Q waves in the anterior and lateral leads \\
\hline & 24 & Left & T-wave inversion in lateral leads \\
\hline & 25 & Left & LV hypertrophy \\
\hline & 26 & Right & First-degree atrioventricular block \\
\hline & 54 & Right & LV hypertrophy \\
\hline \multicolumn{4}{|c|}{ Exercise stress test } \\
\hline & 17 & Right & ST depressions \\
\hline & 20 & Right & ST depressions and chest pain \\
\hline & 33 & Left & ST depressions \\
\hline & 36 & Right & ST depressions \\
\hline & 37 & Right & ST depressions and chest pain \\
\hline & 42 & Right & ST depressions \\
\hline & 47 & Right & ST depressions and chest pain \\
\hline \multicolumn{4}{|c|}{ Nuclear stress test } \\
\hline & 20 & Right & $\begin{array}{l}\text { Small perfusion abnormality in the anteroseptal segment with stress. } \\
\text { Evidence of mild coronary insufficiency }\end{array}$ \\
\hline & 26 & Right & $\begin{array}{l}\text { Moderate ischemia in the distribution of the diagonal or ramus branch of } \\
\text { LAD artery and mild ischemia in distal RCA distribution. }\end{array}$ \\
\hline & 28 & Right & $\begin{array}{l}\text { Small size, mild intensity reversible basal inferior and inferolateral } \\
\text { perfusion defect suggestive of ischemia in the distribution of the RCA } \\
\text { and/or LCx }\end{array}$ \\
\hline & 38 & Left & $\begin{array}{l}\text { Medium-sized, mild defect in the lateral, inferior and posterior region in the } \\
\text { distribution of the RCA and/or LCx }\end{array}$ \\
\hline & 42 & Right & Medium-sized, mild defect \\
\hline & 47 & Right & Mild to moderate basal lateral ischemia without infarction \\
\hline & 54 & Right & Moderate, predominantly reversible defects in the basal to mid inferior wall \\
\hline
\end{tabular}

$E F$, Ejection fraction; $L V$, left ventricular; $R V$, right ventricular; $T R$, tricuspid regurgitation; $L A D$, left anterior descending; $R C A$, right coronary artery; $L C x$, left circumflex artery 